\title{
Rede de atenção às urgências: avanços e desafios para sua consolidação
}

\section{Network of attention to the er: advances and challenges to its consolidation}

\section{Red de atención a emergencias: avances y desafíos para su consolidación}

Carla Pintas MARQUES ${ }^{1}$

Resumo: O texto aborda as definições utilizadas para rede de atenção à saúde e como esta se encontra estabelecida no Sistema Único de Saúde além de contextualizar a atual crise contemporânea dos sistemas de saúde. Aborda também a organização proposta para a consolidação da rede de atenção às urgências como um grande desafio ao sistema de saúde brasileiro.

Descritores: Atenção à Saúde, Emergências, Sistemas de Saúde

Summary: The text discusses the definitions used for network of health care and how this is established in the unified Health System in addition to contextualize the current contemporary crisis of healthcare systems. Also addresses the organization proposal for consolidation of the network of attention to the emergency room as a great challenge to the Brazilian health system.

Descriptors: Health Care, Emergencies, Health Systems

Resumen: El texto analiza las definiciones utilizadas para la red de atención de la salud y cómo esto se establece en el sistema unificado de salud además de contextualizar la crisis contemporánea de los sistemas sanitarios. También aborda la propuesta de la organización para la consolidación de la red de atención a la sala de emergencias como un gran reto para el sistema de salud brasileño. Descriptores: Atención a la Salud, Urgências Médicas, Sistemas de Salud

\section{INTRODUÇÃO}

Os sistemas de atenção à saúde são definidos pela Organização Mundial da Saúde1 como o conjunto de atividades cujo propósito primário é promover, restaurar e manter a saúde de uma população para se atingirem os seguintes objetivos: o alcance de um nível ótimo de saúde, distribuído de forma equitativa; a garantia de uma proteção adequada dos riscos para todos os

1 Mestre em Administração de Saúde pelo Programa de Pós- Graduação em Saúde Coletiva do Instituto de Medicina Social da Universidade Estadual do Rio de Janeiro IMS/UERJ. 
cidadãos; o acolhimento humanizado dos cidadãos; a provisão de serviços seguros e efetivos; e a prestação de serviços eficientes.

Os sistemas de atenção à saúde constituem respostas sociais, deliberadamente organizadas, para responder às necessidades, demandas e preferências das sociedades. Nesse sentido, eles devem ser articulados pelas necessidades de saúde da população que se expressam, em boa parte, em situações demográficas e epidemiológicas singulares ${ }^{2}$.

A categoria condição de saúde é fundamental na atenção à saúde porque, conforme assinalam Porter e Teisberg ${ }^{3}$, só se agrega valor para as pessoas nos sistemas de atenção à saúde quando se enfrenta uma condição de saúde por meio de um ciclo completo de atendimento a ela.

A crise contemporânea dos sistemas de atenção à saúde reflete o desencontro entre uma situação epidemiológica dominada relativamente pelas condições crônicas - nos países desenvolvidos de forma mais definitiva e no Brasil pela situação de tripla carga de doenças - e um sistema de atenção à saúde voltado para responder às condições agudas ${ }^{2,4}$.

Mendes4 nos mostra que em uma observação dos sistemas de atenção à saúde, numa perspectiva internacional, estes são dominados pelos sistemas fragmentados voltados para a atenção às condições agudas e que se (des) organizam a partir de um conjunto de pontos de atenção à saúde isolados, sem comunicação entre eles e, por consequência, incapazes de prestar atenção contínua à população.

A solução do problema fundamental do Sistema Único de Saúde (SUS) consiste em restabelecer a coerência entre a situação de saúde de tripla carga de doenças com predominância de condições crônicas e o sistema de atenção à saúde. Isso vai exigir mudanças profundas que permitam superar o sistema fragmentado vigente a partir da implantação de redes de atenção à saúde ${ }^{2,4}$.

\section{REDES DE ATENÇÃO À SAÚDE (RAS)}

Para Castells ${ }^{5}$, as redes são novas formas de organização social, do Estado ou da sociedade, intensivas em tecnologia de informação e baseadas na cooperação entre unidades dotadas de autonomia. Diferentes conceitos coincidem em elementos comuns das redes: relações relativamente estáveis, autonomia, inexistência de hierarquia, compartilhamento de objetivos comuns, cooperação, confiança, interdependência e intercâmbio constante e duradouro de recursos.

Para Mendes $^{2}$ as propostas de redes no suporte às políticas públicas têm sido crescentemente, adotadas, a partir da década de 90, para superar o modelo burocrático e hierárquico hegemônico, num contexto de complexificação das questões sociais, de processos de privatização, de descentralização acelerada, de globalização, de proliferação de organizações não governamentais e de fortalecimento do controle público. As organizações hierárquicas rígidas caracterizadas por 
pirâmides hierárquicas e por um modo de produção ditado pelos princípios do taylorismo e do fordismo tendem a serem substituídos por redes estruturadas em tessituras flexíveis e abertas de compartilhamentos e interdependências em objetivos, informações, compromissos e resultados.

As redes têm sido propostas para administrar políticas e projetos em que os recursos são escassos e os problemas complexos; onde há interação de agentes públicos e privados, centrais e locais; onde se manifesta uma crescente demanda por benefícios e por participação cidadã6. A gestão eficaz das redes implica: trabalhar rotineiramente na produção de consensos; operar com situações em que todos os atores ganhem; harmonizar os decisores políticos e administrativos; negociar as soluções; e monitorar e avaliar permanentemente os processos ${ }^{7}$.

AOrganização Mundial da Saúde9 considera que as RASs contêm seis modalidades de integração: um conjunto amplo de intervenções preventivas e curativas para uma população; os espaços de integração de vários serviços; a atenção à saúde contínua, ao longo do tempo; a integração vertical de diferentes níveis de atenção; a vinculação entre a formulação da política de saúde e a gestão; e o trabalho intersetorial. A partir dessas modalidades produz uma conceituação de serviços integrados de saúde como "a gestão e a oferta de serviços de saúde de forma a que as pessoas recebam um contínuo de serviços preventivos e curativos, de acordo com as suas necessidades, ao longo do tempo e por meio de diferentes níveis de atenção à saúde".

A Organização Pan-Americana da Saúde10 sugere que as redes integradas de serviços de saúde requerem alguns atributos para seu funcionamento: a população/ território definida, com amplo conhecimento de suas necessidades e preferências em serviços de saúde que devem determinar o perfil de oferta das redes de atenção à saúde; a oferta extensa de serviços de saúde que incluam intervenções de saúde pública, de promoção da saúde, de prevenção das doenças, de diagnóstico e tratamento oportunos, de reabilitação e de cuidados paliativos, sob um único guarda-chuva institucional; a atenção primária à saúde que atua, de fato, como porta de entrada do sistema de atenção à saúde, que integra e coordena a atenção à saúde e que resolve a maioria das necessidades de saúde da população; a prestação de serviços especializados nos lugares apropriados, especialmente em ambientes extra-hospitalares; a existência de mecanismos de coordenação da atenção ao longo de todo o contínuo de cuidados; a atenção centrada nas pessoas, nas famílias e na comunidade; o sistema de governança participativo e único para toda a rede de atenção à saúde; a gestão integrada dos sistemas administrativos e da clínica; os recursos humanos suficientes, competentes e comprometidos com as redes de atenção à saúde; o sistema de informação integrado e que vincula todos os componentes das redes integradas de serviços de saúde; o financiamento adequado e os incentivos financeiros alinhados com os objetivos das redes integradas de serviços de saúde; e a ação inter setorial ampla. Com base nesses atributos conceitua as redes integradas de serviços de saúde como "uma rede de organizações que presta, ou faz arranjos para prestar, serviços de saúde equitativos e integrais a uma população definida e que está disposta a prestar contas por seus resultados clínicos e econômicos e pelo estado de saúde da população a que serve'. 
Com isso em 2010, o Ministério da Saúde10 publica normatização onde define Redes de Atenção à Saúde como arranjos organizativos de ações e serviços de saúde, de diferentes densidades tecnológicas, que, integrados por meio de sistemas de apoio técnico, logístico e de gestão, buscam garantir a integralidade do cuidado. Caracterizam-se pela formação de relações horizontais entre os pontos de atenção, sendo a Atenção Primária à Saúde o centro de comunicação.

\section{REDE DE ATENÇÃO ÀS URGÊNCIAS (RAU)}

Observa-se, em todos os países, um aumento constante na demanda por serviços de urgência e uma consequente pressão, muitas vezes insuportável, sobre as estruturas e os profissionais de saúde. A urgência é a principal causa de insatisfação da população que utiliza o sistema de atenção à saúde4. Sempre haverá uma demanda por serviços maior que a oferta e o aumento da oferta sempre acarreta aumento da demanda, criando-se, assim, um sistema de difícil equilíbrio. A solução tem sido a construção de alternativas de racionalização da oferta ou estratégias regulatórias ${ }^{2,4}$.

Entre as redes de atenção prioritárias do Ministério da Saúde, a RAU foi constituída considerando que o atendimento aos usuários com quadros agudos deve ser prestado por todas as portas de entrada dos serviços de saúde do SUS, possibilitando a resolução integral da demanda ou transferindo-a, responsavelmente, para um serviço de maior complexidade, dentro de um sistema hierarquizado e regulado, organizado em redes regionais de atenção às urgências enquanto elos de uma rede de manutenção da vida em níveis crescentes de complexidade e responsabilidade ${ }^{11}$.

A organização da RAU tem a finalidade de articular e integrar no âmbito do SUS todos os equipamentos de saúde, objetivando ampliar e qualificar o acesso humanizado e integral aos usuários em situação de urgência nos serviços de saúde de forma ágil e oportuna, e deve ser implementada gradativamente, em todo o território nacional, respeitando-se os critérios epidemiológicos e de densidade populacional ${ }^{11}$.

Constituem a base do processo e dos fluxos assistenciais de toda a RAU o acolhimento com classificação do risco, a qualidade e a resolutividade na atenção, que devem ser requisitos de todos os pontos de atenção.

O objetivo da classificação de risco é definir não um diagnóstico, mas uma prioridade clínica, facilitando a gestão da clínica e a gestão do serviço. $\mathrm{O}$ diagnóstico não está ligado à prioridade. $\mathrm{O}$ método requer que o profissional defina o motivo pelo qual a pessoa usuária procura o serviço de urgência, a queixa principal. Segue-se a escolha, entre as condições apresentadas em fluxogramas, daquele mais pertinente à queixa. Depois, procura-se um conjunto de sinais e sintomas em cada nível de prioridade clínica, os discriminadores, nos fluxogramas de apresentação, compatível com a gravidade apresentada ${ }^{12}$. 
A integração dos serviços de urgência e emergência em rede pode determinar um fluxo pactuado pelos serviços. O sistema de classificação de risco é potente ferramenta de comunicação, coresponsabilização e regulação, nas RASs, das condições agudas para acompanhar em tempo real os fluxos e regular o transporte entre os serviços2,4. E a atenção no lugar certo e com a qualidade certa, permite retirar dos pontos de atenção de maior densidade tecnológica, a maioria das pessoas que se apresentam em situações de urgência, os azuis e os verdes, atendendo-os prioritariamente na atenção primária à saúde. Isso é uma condição necessária para superar os graves problemas que ocorrem nos hospitais de urgência e emergência superlotados, com pessoas em macas nos corredores, filas enorme e que estão permanentemente na mídia nacional.

A organização das redes de atenção às urgências e emergências faz-se segundo os seguintes critérios: utilização de protocolo único de classificação de risco; fluxos de encaminhamento, após a classificação dos riscos, desenhados e pactuados internamente para cada instituição e responsabilizados nominalmente pelos respectivos trabalhadores, com descrição e aceitação dos papéis propostos; discussão do fluxo de encaminhamento de responsabilização coletiva, assinada por todos que se co-responsabilizam pelos serviços dessa rede; pactuação dos fluxos de encaminhamento pós-classificação de risco entre todos os atores dos serviços, de todos os níveis de atenção, compartilhada por uma estrutura reguladora também responsável pelo transporte da pessoa usuária, se necessário, até o destino pactuado; compartilhamento das regras com as estruturas de logística (regulação, Serviço de Atendimento Móvel de Urgência (SAMU 192), prontuário eletrônico) que se co-responsabilizam pelos resultados; e informatização dos processos ${ }^{12}$.

No desenho elaborado pelo Ministério da Saúde ${ }^{11}$ para composição da RAU, foram estabelecidos os seguintes componentes:

- Promoção, Prevenção e Vigilância à Saúde: objetiva estimular e fomentar o desenvolvimento de ações de saúde e educação permanente voltadas para a vigilância e prevenção das violências e acidentes, das lesões e mortes no trânsito e das doenças crônicas não transmissíveis, além de ações intersetoriais, de participação e mobilização da sociedade, visando à promoção da saúde, prevenção de agravos e vigilância à saúde.

- Atenção Básica em Saúde: objetiva a ampliação do acesso, fortalecimento do vínculo e responsabilização e o primeiro cuidado às urgências e emergências, em ambiente adequado, até a transferência/ encaminhamento a outros pontos de atenção, quando necessário, com a implantação de acolhimento com avaliação de riscos e vulnerabilidades.

- SAMU 192 e suas Centrais de Regulação Médica das Urgências: objetiva chegar precocemente à vítima após ter ocorrido um agravo à sua saúde (de natureza clínica, cirúrgica, traumática, obstétrica, pediátrica, psiquiátrica, entre outras) que possa levar a sofrimento, sequelas ou mesmo à morte, sendo necessário garantir atendimento e/ou transporte adequado para um serviço de saúde 
devidamente hierarquizado e integrado ao SUS.

- Sala de Estabilização: objetiva funcionar como local de assistência temporária para estabilização de pacientes críticos/graves, vinculado a um equipamento de saúde, articulado e conectado aos outros níveis de atenção, para posterior encaminhamento à Rede de Atenção à Saúde pela Central de Regulação das Urgências.

- Força Nacional de Saúde do SUS: objetiva aglutinar esforços para garantir a integralidade na assistência em situações de risco ou emergenciais para populações com vulnerabilidades específicas e/ou em regiões de difícil acesso, pautando-se pela equidade na atenção, considerando-se seus riscos.

- Unidades de Pronto-Atendimento (UPA 24h) e o Conjunto de Serviços de Urgência 24 horas: objetiva prestar atendimento resolutivo e qualificado aos pacientes acometidos por quadros agudos ou agudizados de natureza clínica e prestar primeiro atendimento aos casos de natureza cirúrgica ou de trauma, estabilizando os pacientes e realizando a investigação diagnóstica inicial, definindo, em todos os casos, a necessidade ou não de encaminhamento a serviços hospitalares de maior complexidade.

- Atenção Hospitalar: objetiva organizar a atenção às urgências nos hospitais, atendendo à demanda espontânea e/ou referenciada, e funcionar como retaguarda para os outros pontos de atenção às urgências de menor complexidade. É constituído pelas Portas Hospitalares de Urgência, pelas enfermarias de retaguarda clínicas e de longa permanência, pelos leitos de cuidados intensivos e pela reorganização das linhas de cuidados prioritárias: Cardiologia - Infarto Agudo do Miocárdio - IAM, Neurologia e Neurocirurgia - Acidente Vascular Cerebral - AVC e Traumatologia.

- Atenção Domiciliar: objetiva a reorganização do processo de trabalho das equipes que prestam cuidado domiciliar na atenção básica, ambulatorial e hospitalar, com vistas à redução da demanda por atendimento hospitalar e/ou redução do período de permanência de pacientes internados, a humanização da atenção, a desinstitucionalização e a ampliação da autonomia dos usuários.

\section{CONSIDERAÇÕES FINAIS}

No Brasil, o atendimento às urgências mostra deficiências estruturais do sistema de saúde, tais como: dificuldades de acesso em vários níveis de atenção, insuficiência de leitos especializados, incipiência dos mecanismos de referência e inadequação na formação dos profissionais de saúde. A implantação das RAS's convoca mudanças radicais no modelo de atenção à saúde praticada no SUS e aponta para a necessidade da implantação de novos modelos de atenção às condições agudas e crônicas. 
É fundamental melhorar a qualidade e a gestão da atenção prestada à população pelas portas de entrada prioritárias da RAU, no âmbito do SUS, identificadas como relevantes na atenção às urgências. Aprimorar os processos de gestão e os investimentos de recursos de saúde com maior qualidade em todas as instituições causa impacto na reorganização dos serviços e promovem a mudança que se deseja no modelo de atenção à saúde.

\section{REFERÊNCIAS BIBLIOGRÁFICAS}

1. World Health Organization. The world health report 2000: health systems, improving performance. Geneva, World Health Organization, 2000.

2. Mendes, EV. As redes de atenção à saúde. Brasília: Organização Pan-Americana da Saúde, 2011. 549p.

3. Porter, ME, Teisberg, EO. Repensando a saúde: estratégias para melhorar a qualidade e reduzir os custos. Porto Alegre, Bookman Companhia Editora, 2007.

4. Mendes, EV. As Redes de Atenção à Saúde. Rev Med Minas Gerais 2008; 18(4 Supl 4): S3S11

5. Castells, M. A sociedade em rede. São Paulo, Paz e Terra, Volume I, 4ª ed., 2000.

6. Fleury, SMT, Ouverney, AM. Gestão de redes: a estratégia de regionalização da política de saúde. Rio de Janeiro, Editora FGV, 2007.

7. Agranoff, R., Lindsay, VA. Intergovernmental management: perspectives from human services problem solving at the local level. Public Administration Review, 43: 227-238, 1983.

8. World Health Organization. Integrated health services: what and why? Geneva, World Health Organization, Technical Brief $\mathrm{n}^{\mathrm{o}}$ 1, 2008.

9. Organización Panamericana de la Salud. Redes integradas de servicios de salud: conceptos, opciones de politica y hoja de ruta para su implementación en las Américas. Washington, HSS/IHS/ OPS, Serie La Renovación de la Atención Primaria de Salud en las Américas, 2010.

10. Brasil. Portaria MS/GM N 4.279, de 30 de dezembro de 2010. Disponível em: http:// bvsms. saude.gov.br/bvs/saudelegis/gm/2010/prt4279_30_12_2010.html. Acesso em 15 de junho de 2013. 
11. Brasil. Portaria MS/GM N 1.600, de 7 de julho de 2011. Disponível em: http://bvsms.saude. gov.br/ bvs/saudelegis/gm/2011/prt1600_07_07_2011.html. Acesso em 15 de junho de 2013.

12. Cordeiro Júnior, W, Mafra, A de A. A rede de atenção à urgência e emergência e o protocolo de classificação de risco de Manchester. Belo Horizonte, Secretaria de Estado de Saúde de Minas Gerais, 2008.

Artigo apresentado em 12/01/13

Artigo aprovado em 15/03/14

Artigo publicado no sistema: 25/03/14 